\title{
Helium-star evolutionary channel to super-Chandrasekhar mass type la supernovae
}

\author{
W.-M. Liu ${ }^{1}$, W.-C. Chen ${ }^{1,2,3}$, B. Wang ${ }^{4}$, and Z. W. Han ${ }^{4,5}$ \\ 1 Department of Physics, Shangqiu Normal University, Shangqiu 476000, PR China \\ e-mail: chenwc@nju.edu.cn \\ 2 School of Physics and State Key Laboratory of Nuclear Physics and Technology, Peking University, Beijing 100871, PR China \\ 3 Key Laboratory of Modern Astronomy and Astrophysics (Nanjing University), Ministry of Education, Nanjing 210093, PR China \\ ${ }^{4}$ National Astronomical Observatories/Yunnan Observatory, the Chinese Academy of Sciences, Kunming 650011, PR China \\ 5 Key Laboratory for the Structure and Evolution of Celestial Objects, the Chinese Academy of Sciences, Kunming 650011, PR China
}

Received 2 February 2010 / Accepted 27 July 2010

\begin{abstract}
The recent discovery of several overluminous type Ia supernovae (SNe Ia) indicates that the explosive masses of white dwarfs may significantly exceed the canonical Chandrasekhar-mass limit. Rapid differential rotation may support these massive white dwarfs (WDs). Based on the single-degenerate scenario and assuming that the WDs would differentially rotate when the accretion rate $\dot{M}>3 \times 10^{-7} M_{\odot} \mathrm{yr}^{-1}$, we performed the numerical calculations for $\sim 1000$ binary systems consisting of a He star and a CO WD with Eggleton's stellar evolution code. We present the initial parameters in the orbital period - helium star-mass plane (for WD masses of $1.0 M_{\odot}$ and $1.2 M_{\odot}$, respectively), which lead to super-Chandrasekhar mass SNe Ia. Our results indicate that for an initially massive WD of $1.2 M_{\odot}$, a large number of SNe Ia may result from super-Chandrasekhar mass WDs. The highest mass of the WD at the moment of the SNe Ia explosion is $1.81 M_{\odot}$, but very massive $\left(>1.85 M_{\odot}\right)$ WDs cannot be formed. However, when the initial mass of WDs is $1.0 M_{\odot}$, the explosive masses of SNe Ia are nearly uniform, which is consistent with the rareness of super-Chandrasekhar mass $\mathrm{SNe}$ Ia in observations.
\end{abstract}

Key words. binaries: general - stars: rotation - stars: evolution - supernovae: general - white dwarfs

\section{Introduction}

Type Ia supernovae ( $\mathrm{SNe}$ Ia) are generally believed to be the thermonuclear explosions of carbon-oxygen white dwarfs (CO WDs) in binaries (for a review see Nomoto et al. 1997). Because of a uniform luminosity, $\mathrm{SNe}$ Ia are used as the standard candlelight to determine the cosmological distances and estimate the cosmological parameters $\Omega$ and $\Lambda$ (e.g. Riess et al. 1998; Perlmutter et al. 1999). However, some key issues including the properties of their progenitors and the physical mechanisms of the explosion are still poorly known by astrophysicists (Hillebrandt \& Niemeyer 2000; Röpke \& Hillebrandt 2005; Wang et al. 2008; Podsiadlowski et al. 2008).

At present, there exist two progenitor models of SNe Ia, i.e. single-degenerate model (Whelan \& Iban 1973; Nomoto 1982) and a double-degenerate model (Iben \& Tutukov 1984; Webbink 1984). Over the past decade, many groups have widely investigated the single-degenerate model, in which the CO WD accretes $\mathrm{H} / \mathrm{He}$-rich material from a non-degenerate companion star. In this scenario, the donor star of WD may be a mainsequence star, a subgiant star or a red-giant star (see Hachisu et al. 1996; Li \& van den Heuvel 1997; Hachisu et al. 1999a,b; Langer et al. 2000; Han \& Podsiadlowski 2004, 2006; Chen \& Li 2007; Han 2008; Chen \& Li 2009; Meng et al. 2009; Lü et al. 2009; Wang et al. 2010; Meng \& Yang 2010a,b).

Limongi \& Tornambe (1991) were the first to study the evolution of the binary consisting of a CO WD and a He star, in which the CO WD accretes He matter from the He star and gains mass up to the canonical Chandrasekhar limit of $1.4 M_{\odot}$.
Yoon \& Langer (2003) also found that CO WD + He star systems can form a reliable channel that produces SNe Ia. Recently, Wang et al. (2009a) systematically explored the evolution of 2600 close WD binaries that include a He MS star or He subgiant, and obtained the parameter spaces for the progenitor of SNe Ia. With a detailed binary population synthesis approach, Wang et al. (2009b) suggested that this channel can contribute a SN Ia birthrate of $3 \times 10^{-4} \mathrm{yr}^{-1}$ in the Galaxy, and can result in the short delay times ( $\$ 100 \mathrm{Myr})$ between the formation of the progenitor systems and the explosions.

Recently, the overluminous SN Ia 2003fg has been detected (Astier et al. 2006), and its explosive mass was estimated to be $\sim 2.1 M_{\odot}$ (Howell et al. 2006) ${ }^{1}$. Later, several possible overluminous SNe Ia 2006gz, 2007if, and 2009dc were also discovered (Hicken et al. 2007; Yuan et al. 2007; Tanaka et al. 2010; Scalzo et al. 2010; Silverman et al. 2010). The differential rotation may support these massive WDs (Yoon et al. 2004; Yoon \& Langer 2004; Yoon \& Langer 2005), which may also result from the merger of two massive WDs (Tutukov \& Yungelson 1994; Howell 2001; Piersanti et al. 2003). Based on the single degenerate model and the assumption that the WD rotates differentially, Chen \& Li (2009) have explored the evolution of close binaries consisting of a WD and a $\mathrm{H}$ main sequence star. Their results indicate that for an initial massive WD with $1.2 M_{\odot}$, the maximum explosive mass of the WDs is $1.76 M_{\odot}$.

1 Hillebrandt et al. (2007) suggested that a lopsided explosion due to the off-center ignition of nuclear burning in a WD with Chandrasekhar mass may be responsible for the high brightness of $2003 \mathrm{fg}$. 
It is an interesting issue to explore the progenitors of overluminous SNe Ia based on the single-degenerate model. In this paper, we attempt to explore whether the WD + He star systems can produce overluminous SNe Ia. To obtain the distribution of the initial He star mass and the initial orbital period of the progenitor binaries, we have calculated the evolution of $1000 \mathrm{WD}$ binaries that include a He star and a rotating CO WD. In Sect. 2 we give a detailed description of the input physics including the formation of WD + He star systems and the binary evolution code. In Sect. 3 we present the calculated results. Finally, a brief discussion and summary are presented.

\section{Input physics}

\subsection{Formation of $W D+$ He star systems}

In young stellar populations with relatively late star-formation exist intermediate-mass binary systems, which can evolve into $\mathrm{CO}$ WD + He star systems. For a large range of initial parameters, close binaries can evolve into a system consisting of a more massive WD and a less massive He star (Tornambè \& Matteucci 1986; Iben \& Tutukov 1987; Iben et al. 1987). The mass-transfer rate in these systems is only $\sim 3 \times 10^{-8} M_{\odot} \mathrm{yr}^{-1}$, which may result in an explosion of SN magnitude after the WD accumulates $\sim 0.15 M_{\odot}$ of helium (Iben \& Tutukov 1991). However, Iben \& Tutukov (1994) proposed that WD + massive He star systems can be formed via binary evolution, and the He shell burning on the surface of the CO WD could be in a quasi-stationary after the massive He star fills its Roche lobe.

Using the rapid binary star evolution (BSE) code (Hurley et al. 2000, 2002), we calculated the formation process of a massive CO WD + He star system. We consider a primordial binary consisting of a primary (star 1$)$ of $M_{1}=7.5 M_{\odot}$ and a star 2 of $M_{2}=4.0 M_{\odot}(Z=0.02)$ and with an orbital separation of $a=50.93 R_{\odot}$. Owing to the nuclear evolution, when $t=42.42 \mathrm{Myr}$ the primary firstly fills its Roche lobe, and transfers its material to the star 2 . Because of a relatively high initial mass ratio, the mass transfer occurs on a short thermal timescale. When $t=42.52 \mathrm{Myr}, M_{1}=1.482 M_{\odot}, M_{2}=10.001 M_{\odot}$, and the first Roche-lobe overflow ceases. When $t=52.20 \mathrm{Myr}$, the primary that evolves into a He star fills its Roche lobe again, and then evolves into a CO WD of $M_{1}=1.103 M_{\odot}$ after the second Roche-lobe overflow ceases. At the same time, the star 2 is in a Hertzsprung gap, and $M_{2}=10.194 M_{\odot}$. When $t=64.33 \mathrm{Myr}$, the star 2 continues to evolve and fills its Roche lobe. Due to the ultra-high mass ratio, a common envelope is possibly quickly formed because of the dynamically unstable mass transfer. After the common envelope is ejected, a close binary system consisting of a CO WD $\left(M_{1}=1.103 M_{\odot}\right)$ and a He $\operatorname{star}\left(M_{2}=2.247 M_{\odot}\right)$ is formed. This formation channel is known as the He star channel (for details see Wang et al. 2009b), which allows stable Roche-lobe overflow to produce massive $\mathrm{WD}+\mathrm{He}$ star systems (rather than lead to dynamical mass transfer and a common envelope phase).

In addition, another two channels such as the early asymptotic giant branch channel and the thermal-pulsing asymptoticgiant branch channel can also produce CO WD + He star systems (Wang et al. 2009b). Employing Hurley's rapid BSE code, Wang et al. (2009b) investigated the initial parameter distribution of WD + He star systems. Their simulated results show that the initial mass of the CO WDs is in the range of $0.85-$ $1.2 M_{\odot}{ }^{2}$, and the initial mass of the He stars is in the range of

\footnotetext{
2 The rapid-binary evolution code presents a rough statistical result, while the standard stellar evolutionary theory suggested that $1.1 \mathrm{M}_{\odot}$
}

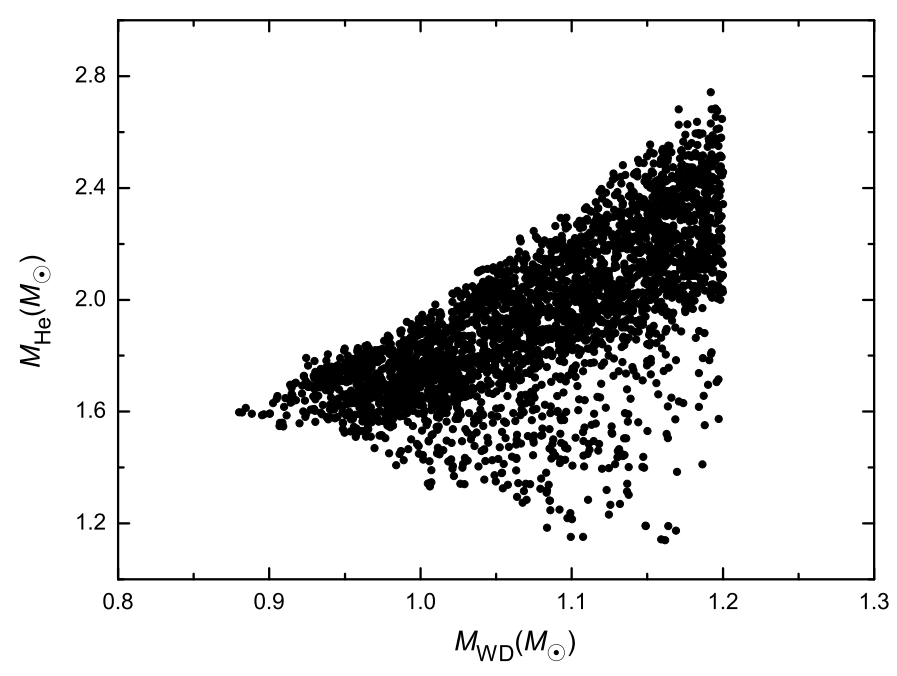

Fig. 1. Distribution of initial WD + He star systems in $M_{\mathrm{He}}-M_{\mathrm{WD}}$ diagram. Basic input parameters for Monte Carlo simulations correspond to the standard model of Wang et al. (2009b).

1.0-2.8 $M_{\odot}$, which depends on the common-envelope parameters $\alpha_{\mathrm{CE}} \lambda$. Figure 1 presents the distribution of the initial WD + He star systems in $M_{\mathrm{He}}-M_{\mathrm{WD}}$ diagram, in which we have followed the evolution of $4 \times 10^{7}$ sample binaries from the star formation to the formation of the WD $+\mathrm{He}$ star systems and adopt $\alpha_{\mathrm{CE}} \lambda=0.5$. Based on this work, for input parameters we take the initial mass of the WDs $M_{\mathrm{WD}, i}=1.0,1.2 M_{\odot}$, the initial mass of the He stars $M_{\mathrm{He}, i}=1.0-2.8 M_{\odot}$, and the initial orbital period $\log \left(P_{\mathrm{orb}, i} / d\right)=-1.4-0$ (see Fig. 3 in Wang et al. 2009b).

\subsection{Binary evolution code}

Employing the stellar evolution code developed by Eggleton (see Eggleton 1971, 1972, 1973), which has been updated with the latest input physics (Han et al. 1994; Pols et al. 1995, 1998), we study the evolution of close binaries consisting of a rotating CO WD (of mass $M_{\mathrm{WD}}$ ) and a He star (of mass $M_{\mathrm{He}}$ ). The stellar OPAL opacities come from Rogers \& Iglesias (1992) and Alexander \& Ferguson (1994) for a low temperature. In our calculations, we set the ratio of mixing length to local pressure scale height to be 2.0, and the overshooting parameter of the He stars (with chemical abundance $Y=0.98, Z=0.02$ ) is taken to be 0 (Dewi et al. 2002).

In a WD + He star system, due to the nuclear evolution or the loss of orbital angular momentum the He star fills its Roche lobe at He main sequence or He subgiant stage, and then begins the mass transfer. The He-rich material from the He star is

is the maximum mass of the $\mathrm{CO}$ core. Assuming that the envelope of AGB star is lost when the binding energy of the envelope is equal to zero, Han et al. (1994) and Meng et al. (2008) found that the maximum mass of CO WD is $1.1 M_{\odot}$ and $1.05 M_{\odot}(Z=0.02)$, respectively. If a CO core with $1.2 M_{\odot}$ is produced by stellar evolution, a C burning should occur and result in the formation of ONeMg WD. Therefore, it is very difficult to form a $1.2 M_{\odot} \mathrm{CO}$ WD from a stellar evolutionary channel. Certainly, the maximum mass of the CO WD is related to the definition of the core, and the necessary condition ejecting the envelope of an AGB star. In addition, the lifting effect caused by rotation may help in producing larger $\mathrm{CO}$ core masses. We expect additional detailed studies to confirm if the most massive CO WD can exceed $\sim 1.1 M_{\odot}$ as a result of binary interaction. Similar to Hachisu et al. (1996) and Li \& van den Heuvel (1997), in this work we still take a maximum initial mass of $1.2 M_{\odot}$ for WDs. 
accreted by the WD, and is converted into CO heavier elements via thermonuclear burning onto the surface of the WD. In input physics, we adopt an optically thick wind scenario (Kato \& Hachisu 1994; Hachisu et al. 1996) and the description for He mass accumulation efficiency onto the surface of the WD given by Kato \& Hachisu (2004). Firstly, if the mass transfer rate $\left|\dot{M}_{\mathrm{He}}\right|$ is above a critical rate (Nomoto 1982)

$\dot{M}_{\text {cr }}=7.2 \times 10^{-6}\left(M_{\mathrm{WD}} / M_{\odot}-0.6\right) \quad M_{\odot} \mathrm{yr}^{-1}$,

we take that He burning is steady, and the He-rich material on the surface of the WD is converted into $\mathrm{CO}$ elements at a rate $\dot{M}_{\text {cr }}$. Secondly, if $\left|\dot{M}_{\mathrm{He}}\right|$ is lower than $\dot{M}_{\text {cr }}$ but higher than $\dot{M}_{\text {st }}$ (Kato \& Hachisu 2004), which is the minimum accretion rate that the He-shell steadily burns, the He burning is thought to be steady, and all accreting material is converted into $\mathrm{CO}$ elements. Thirdly, if $\left|\dot{M}_{\mathrm{He}}\right|$ is lower than $\dot{M}_{\text {st }}$ but higher than $\dot{M}_{\text {low }}=$ $4.0 \times 10^{-8} M_{\odot} \mathrm{yr}^{-1}$, in which the weak He-shell flashes occur ${ }^{3}$, a part of the envelope material of the WD is assumed to be blown off the surface of the WD (Woosley et al. 1986). Finally, if $\left|\dot{M}_{\mathrm{He}}\right|$ is lower than $\dot{M}_{\text {low }}$, the strong He-shell flashes occur, and no material can be accumulated onto the WD. Summarizing the above prescriptions, the accumulation efficiency of the accreting $\mathrm{He}$ can be written as (Wang et al. 2009a)

$\alpha= \begin{cases}\frac{\dot{M}_{\mathrm{cr}}}{\left|\dot{M}_{\mathrm{He}}\right|}, & \left|\dot{M}_{\mathrm{He}}\right|>\dot{M}_{\mathrm{cr}}, \\ 1, & \dot{M}_{\mathrm{cr}} \geq\left|\dot{M}_{\mathrm{He}}\right| \geq \dot{M}_{\mathrm{st}}, \\ \alpha^{\prime}, & \dot{M}_{\mathrm{st}}>\left|\dot{M}_{\mathrm{He}}\right| \geq \dot{M}_{\mathrm{low}}, \\ 0, & \left|\dot{M}_{\mathrm{He}}\right|<\dot{M}_{\mathrm{low}},\end{cases}$

where $\alpha^{\prime}$ is determined by a linearly interpolated way from a grid computed by Kato \& Hachisu (2004).

Actually, the accumulation efficiency mentioned above is limited to non-rotating WDs and is inadequate to calculate the mass increase of rotating WD. Considering the spin-up of the WDs via accretion, Yoon \& Langer (2004) found that the rotation can stabilize He-shell burning, and help He-accreting CO WD gain mass. Assuming that the CO WDs rotate rigidly, the simulated results by Domínguez et al. (2006) proposed that massive progenitors result in higher ${ }^{56} \mathrm{Ni}$ mass and explosive luminosity, and more massive WDs at the moment of explosion.

To consider the influence of rotation on the mass accumulation of WDs, we adopt the following input physics (e.g. Chen \& Li 2009). (1) With the mass transfer from the He star, the WDs obtain a large amount of angular momentum from the accreting material, and are spun up to a high rotation velocity (Durisen 1977; Ritter 1985; Narayan \& Pophm 1989; Langer et al. 2000). (2) Considering the lifting effect in the hydrostatic equilibrium (Domínguez et al. 1996), we introduce an effective mass $M_{\mathrm{eff}}$ of the WD by taking account of the centrifugal force. (3) According to different ranges of the polar angle, we divide the surface of the WD into three zones as follows: the equatorial zone (EZ, $\theta=60^{\circ}-120^{\circ}$ ), the middle zone (MZ, $\theta=30^{\circ}-60^{\circ}$ and $120^{\circ}-$ $\left.150^{\circ}\right)$, and the polar zone (PZ, $\theta=0^{\circ}-30^{\circ}$ and $\left.150^{\circ}-180^{\circ}\right)$. (4) Assuming that each zone accretes the transferred material at a rate proportional to its area, we can obtain an accretion fraction $f_{i}=0.5,0.366$, and 0.134 for EZ, MZ, and PZ, respectively. Therefore, the mass growth rate of the rotating WD is given by ${ }^{4}$

$\dot{M}_{\mathrm{WD}}=\sum \alpha_{i} f_{i}\left|\dot{M}_{\mathrm{He}}\right|$

\footnotetext{
3 When weak He-shell flashes occur, the mass accumulation efficiency strongly depend on the chemical composition or the radius of the WD (Kato \& Hachisu 2004).

${ }^{4}$ In this work, we have not calculated the detailed evolution of the WD, and only consider the mass accumulation process on the surface.
}

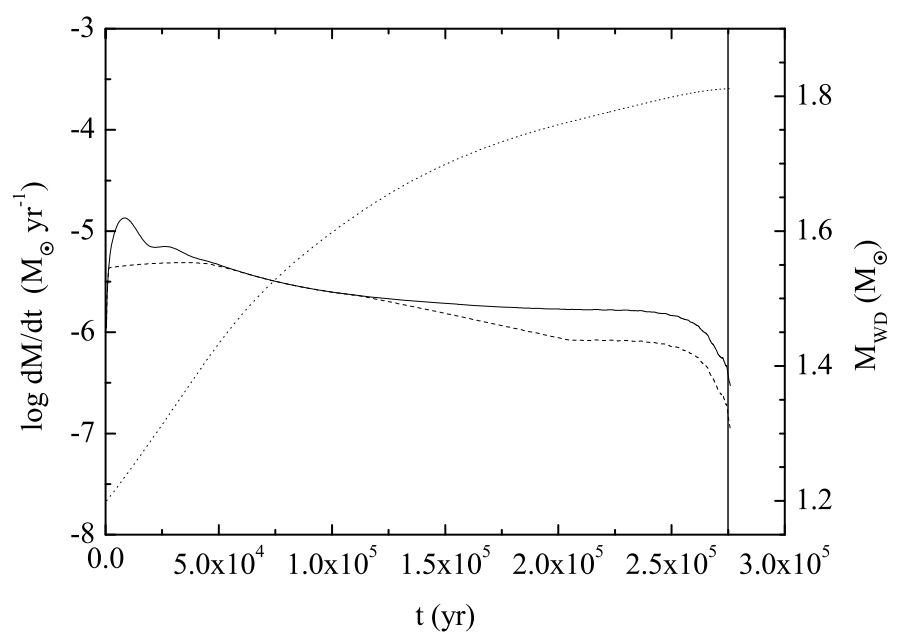

Fig. 2. Evolution tracks of WD binary with $M_{\mathrm{He}, i}=1.8 M_{\odot}, M_{\mathrm{WD}, i}=$ $1.2 M_{\odot}$, and $\log \left(P_{\mathrm{orb}, i} /\right.$ day $)=-1.2$. The solid, dashed, and dotted curves represent the evolution of $\dot{M}_{\mathrm{He}}, \dot{M}_{\mathrm{WD}}$, and $M_{\mathrm{WD}}$, respectively. The solid vertical line indicates the position where the WD is expected to explode as a SN Ia.

where $\alpha_{\mathrm{i}}$ is the mass accumulation efficiency for different zones on the surface of the WD. The mass-loss rate of the binary system is $\dot{M}=\left(1-\sum \alpha_{i} f_{i}\right) \dot{M}_{\mathrm{He}}$, which is assumed to be ejected in the vicinity of the WD in the form of isotropic winds or outflows, and taking away the specific orbital angular momentum of the WD.

In our calculations, we set the initial surface velocity at the WD's equator to be $10 \mathrm{~km} \mathrm{~s}^{-1}$, and the radius of the WD changes with $R \propto M_{\mathrm{WD}}^{-1 / 3}$. The criterion that the WD rotates differentially is a key input physics in this work. We adopt the conclusion derived by Yoon \& Langer (2004), in which the WD should rotate differentially when its accretion rate $\gtrsim 3 \times 10^{-7} M_{\odot} \mathrm{yr}^{-1}$. As a result of differential rotation, the central carbon ignition of the WD cannot occur even if its mass exceeds the canonical Chandrasekhar limit of $1.4 M_{\odot}$. Once $M_{\mathrm{WD}} \geq 1.4 M_{\odot}$ and $\dot{M}<3 \times 10^{-7} M_{\odot}$, we stop the calculation and assume the WD to explode as a SN Ia (we use $M_{\mathrm{SN}}$ to denote the explosive mass of the WD) because there is no more differential rotation to support the massive WD. Considering the prescriptions above for the mass accumulation on the surface of the WD in Eggleton's stellar evolution code, we have calculated the evolution of WD + He star systems, and obtained the initial parameters of the WD binaries that lead to $\mathrm{SNe}$ Ia.

\section{Binary evolution results}

An example of the evolutionary sequences of a WD binary (with $M_{\mathrm{He}, i}=1.8 M_{\odot}, M_{\mathrm{WD}, i}=1.2 M_{\odot}$, and $\log \left(P_{\text {orb }, i} /\right.$ day $\left.)=-1.20\right)$ are shown in Figs. 2 and 3. We plot the evolution of $\dot{M}_{\mathrm{He}}, \dot{M}_{\mathrm{WD}}$ and $M_{\mathrm{WD}}$ varying with time in Fig. 2 . When the age of the He star is $1.76 \times 10^{6} \mathrm{yr}$, the Roche-lobe overflow occurs, and the WD accretes the material from the He star. In the earlier phase of the mass transfer, the orbital period decreases until $\log \left(P_{\text {orb }, i} /\right.$ day $\approx$ -1.24 , because the material is transferred from the more massive He star to the less massive WD, and then increases when the WD mass grows and exceeds the He star mass. After the mass exchange of $2.75 \times 10^{5} \mathrm{yr}$, the mass transfer rate decline to be $3 \times 10^{-7} M_{\odot} \mathrm{yr}^{-1}$, and the WD cannot differentially rotate and SN Ia explosion is triggered. At the moment of the SN explosion, the WD grows to $1.81 M_{\odot}$, the mass of the He star is $0.96 M_{\odot}$, 


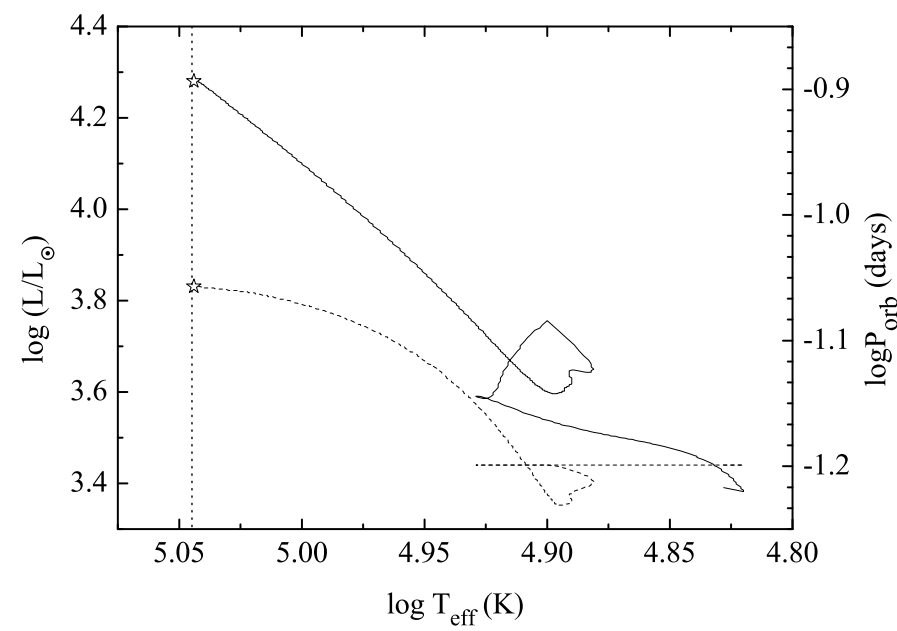

Fig. 3. Evolutionary track of the He star is shown as a solid curve, and the evolution of orbital period is shown as a dashed curve. The vertical dotted line and open stars indicate the place where the WD explodes as a SN Ia.

and $\log \left(P_{\text {orb }} /\right.$ day $)=-1.06$. During the stable He shell burning, the mass-accretion rate of the WD is $10^{-7}-10^{-5} M_{\odot} \mathrm{yr}^{-1}$. By $L_{\mathrm{X}} \sim \epsilon_{\mathrm{He}}\left|\dot{M}_{\mathrm{He}}\right|\left(\epsilon_{\mathrm{He}}=6.0 \times 10^{17} \mathrm{erg} \mathrm{g}^{-1}\right.$ is the released energy rate by He burning), we can estimate the X-ray luminosity of the $\mathrm{WD}+\mathrm{He}$ star system to be $\sim 10^{36}-10^{38} \mathrm{erg} \mathrm{s}^{-1}$. This estimate is consistent with the observed X-ray luminosity for supersoft X-ray sources (SSSs, Kahabka \& van den Heuvel 1997), and confirms the conclusion that WD + He star systems may appear as SSSs before SN Ia explosions (Iben \& Tutukov 1994; Yoon \& Langer 2003).

To obtain the distribution of the initial He star mass and the initial orbital period for the progenitors of super-Chandrasekhar mass SNe Ia, we have calculated the evolutions of about 1000 WD binaries with different input parameters, which include the initial mass of the donor star $M_{\mathrm{He}, i}$, the initial orbital period $P_{\mathrm{orb}, i}$, and the initial mass of the WD $M_{\mathrm{WD}, i}$ (which we take to be $1.2 M_{\odot}$ and $1.0 M_{\odot}$ ). Figure 4 shows our calculation results in $M_{\mathrm{He}, i}-\log P_{\mathrm{orb}, i}$ diagram when $M_{\mathrm{WD}, i}=1.2 M_{\odot}$, and the regions enclosed by the solid, dashed, and dotted curves represent the distribution areas of initial WD binaries with $M_{\mathrm{SN}} \geq$ $1.7 M_{\odot}, M_{\mathrm{SN}} \geq 1.6 M_{\odot}$, and $M_{\mathrm{SN}} \geq 1.4 M_{\odot}$, respectively. Beyond the boundary enclosed by the dotted curve, the mass of the WD cannot reach the Chandrasekhar limit due to either a low mass-accumulation efficiency on the surface of the WD or unstable mass-transfer. In particular, there exist eight WD binaries with an explosive mass of $M_{\mathrm{SN}} \geq 1.8 M_{\odot}$ (see the star signs in Fig. 4), while the maximum explosive mass is $1.76 M_{\odot}$ for WD binaries with a main sequence or sub-giant companion (Chen \& Li 2009). When $M_{\mathrm{WD}, i}=1.0 M_{\odot}$, the progenitors distribution of $\mathrm{SNe}$ Ia in the $M_{\mathrm{He}, i}-\log P_{\mathrm{orb}, i}$ diagram is plotted in Fig. 5, in which the solid and dashed curves represent the region boundaries with $1.5 M_{\odot} \geq M_{\mathrm{SN}} \geq 1.45 M_{\odot}$, and $M_{\mathrm{SN}} \geq 1.4 M_{\odot}$, respectively. In this case, the mass of the WD cannot grow to $\geq 1.5 M_{\odot}$. It is obvious that for a WD with $M_{\mathrm{WD}, i}=1.2 M_{\odot}$, its maximum accumulated mass is greater than those of the WD with $M_{\mathrm{WD}, i}=1.0 M_{\odot}$ by more than $\sim 0.1 M_{\odot}$. This difference mainly originates from the different mass-accumulation efficiency on the surface of the WD. One can see that the higher the WD mass, the higher the critical mass transfer rate, and hence the higher mass-accumulation efficiency under an approximate equal mass transfer rate (see Eqs. (1) and (2)).

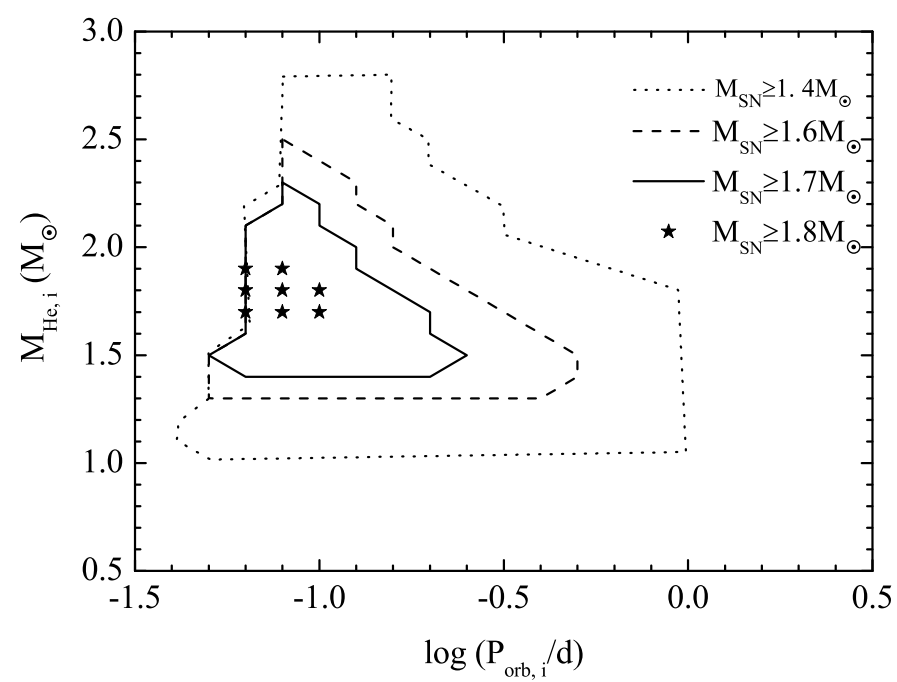

Fig. 4. Distribution of the initial orbital periods $P_{\text {orb }, i}$ and the initial $\mathrm{He}$ star masses $M_{\mathrm{He}, i}$ of the progenitors of super-Chandrasekhar mass SNe Ia when $M_{\mathrm{WD}, i}=1.20 M_{\odot}$.

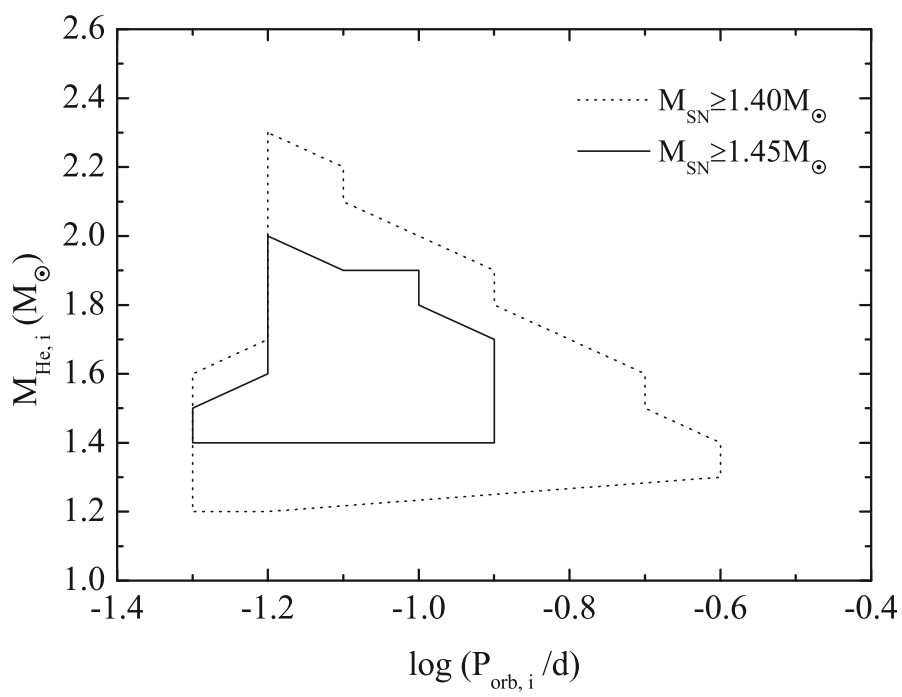

Fig. 5. Similar to Fig. 4, but for an initial WD mass of $1.0 M_{\odot}$.

Figures 6 and 7 present the distribution of the donor stars at the moment of SNe Ia in the H-R diagram when $M_{\mathrm{WD}, i}=$ $1.2 M_{\odot}$, and $M_{\mathrm{WD}, i}=1.0 M_{\odot}$, respectively. They have luminosities in the range of $\log \left(L / L_{\odot}\right) \sim 3.2-4.4$ and effective temperatures in the range of $10000-150000 \mathrm{~K}$. These properties may be compared with and testified by future optical observations of SN Ia remnants.

\section{Discussion and summary}

Overluminous SNe Ia may originate from the thermonuclear explosion of super-Chandrasekhar mass WDs, which may be supported by rapid differential rotation. Inspired by the works of Wang et al. (2009a,b), Wang \& Han (2010a) and Chen \& Li (2009), in this paper we investigated the evolutionary channel of He star + WD with differential rotation, which may be the progenitor of super-Chandrasekhar mass SNe Ia like SN Ia $2003 \mathrm{fg}$. Assuming that the WD rotates differentially when $\dot{M}>3 \times 10^{-7} M_{\odot} \mathrm{yr}^{-1}$, we performed the numerical calculations for $\sim 1000$ WD binaries that consist of a WD and a He star. 


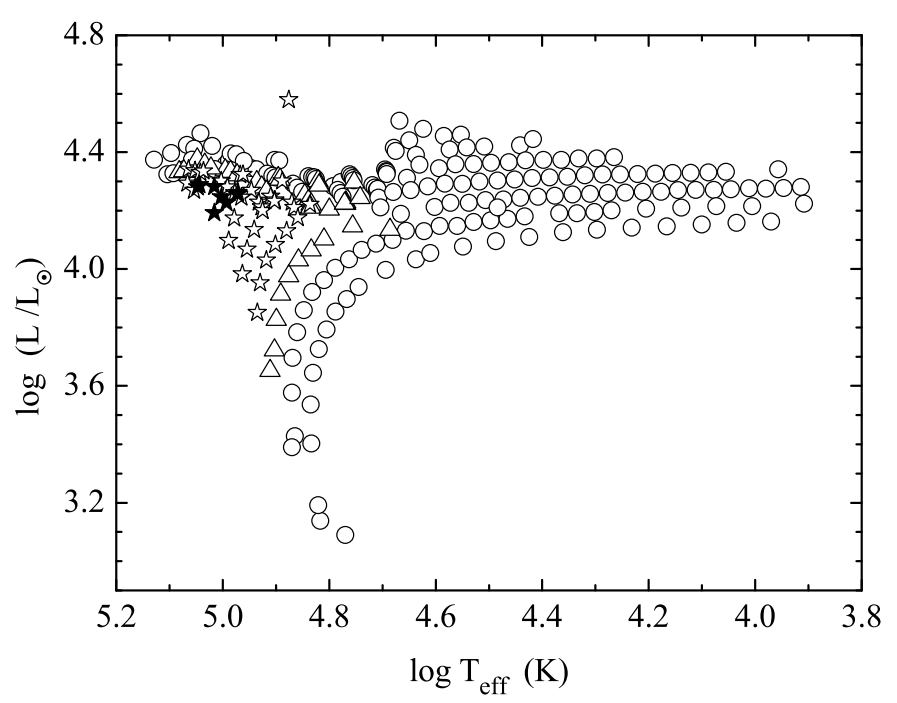

Fig. 6. Distribution of the donor stars at the moment of SN Ia explosions in the H-R diagram when $M_{\mathrm{WD}, i}=1.2 M_{\odot}$. The open circles, open stars, open triangles, and solid stars denote systems with $1.4 M_{\odot} \leq M_{\mathrm{SN}}<$ $1.6 M_{\odot}, 1.6 M_{\odot} \leq M_{\mathrm{SN}}<1.7 M_{\odot}, 1.7 M_{\odot} \leq M_{\mathrm{SN}}<1.8 M_{\odot}$, and $M_{\mathrm{SN}} \geq 1.8 M_{\odot}$, respectively.

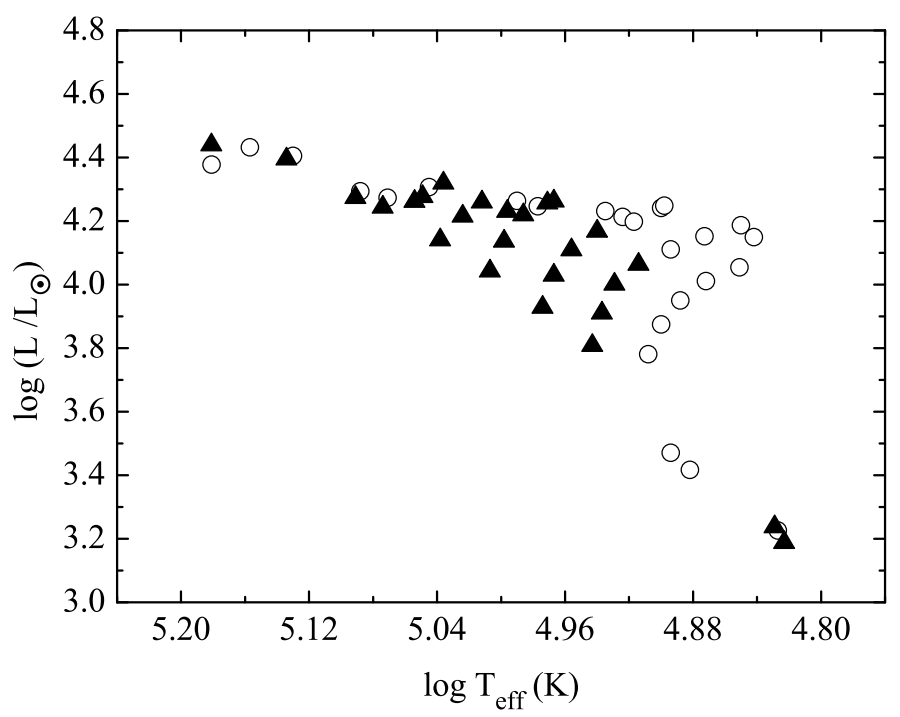

Fig. 7. Similar to Fig. 6, but for $M_{\mathrm{WD}, i}=1.0 M_{\odot}$. The open circles and solid triangles denote systems with $1.40 M_{\odot} \leq M_{\mathrm{SN}}<1.45 M_{\odot}$, and $M_{\mathrm{SN}} \geq 1.45 M_{\odot}$, respectively.

Our main results can be summarized as follows:

1. When the initial mass of the WDs $M_{\mathrm{WD}, i}=1.2 M_{\odot}$, the explosive masses of the WDs are in the range of 1.40-1.81 $M_{\odot}$. This implies that a large number of $\mathrm{SNe}$ Ia may result from super-Chandrasekhar mass WDs, while very massive $\left(M_{\mathrm{SN}}>1.85 M_{\odot}\right)$ WDs cannot be formed. As Chen \& Li (2009), our He-star evolutionary channel cannot produce super-Chandrasekhar mass SN Ia with $M_{\mathrm{SN}} \sim 2.1 M_{\odot}$ like $2003 \mathrm{fg}$ (Howell et al. 2006). It seems that the merger of two massive WDs may be responsible for this peculiar SN Ia (Tutukov \& Yungelson 1994; Howell 2001).

2. When $M_{\mathrm{WD}, i}=1.0 M_{\odot}$, all the explosive masses of the WDs are less than $1.5 M_{\odot}$, i.e. the explosive luminosity of $\mathrm{SNe}$ Ia are nearly uniform. These results are consistent with the rareness of overluminous $\mathrm{SNe} \mathrm{Ia}$ in observations.
3. Compared with the main sequence star, subgiant star, and red-giant channel, the He-star channel has higher explosive masses, less orbital separation, and a shorter mass-transfer timescale.

The WD + He star systems may be the candidate of SSSs. Di Stefano \& Kong (2003) found that some SSSs in spiral galaxies are associated with spiral arms, which shows that they are young systems with an age of $\lesssim 0.1 \mathrm{Gyr}$. The WD + He stars channel can explain SNe Ia with short delay times $\left(\$ 10^{8} \mathrm{yr}\right)$ (Wang et al. 2009a,b; Meng \& Yang 2010b), therefore this channel may be related to the young SSSs. In addition, the WD + He star channel may be responsible for the origin of the hypervelocity stars (Wang \& Han 2009).

The system HD 49798/RXJ 0648.0-418 is confirmation of the existence of a rapidly rotating massive WD + He star system. Based on data from the XMM - Newton satellite, the masses of the hot subdwarf HD 49798 and the WD are constrained to be $1.50 \pm 0.05 M_{\odot}$ and $1.28 \pm 0.05 M_{\odot}$, respectively (Mereghetti et al. 2009). Wang \& Han (2010c) suggested that the hot subdwarf HD 49798 and its X-ray pulsating companion could evolve into an SN Ia in the future. The system V445 Pup is another candidate of a massive WD + He star system, in which the mass of the WD is $\gtrsim 1.35 M_{\odot}$, and the mass of the He star is $\gtrsim 0.85 M_{\odot}$ (Kato et al. 2008; Woudt et al. 2009).

The formation mechanism of the overluminous $\mathrm{SNe}$ Ia is still an open question. Both the merger of two massive WDs and the differentially rotating WDs in a close binary may be the progenitors of overluminous SNe Ia. For the latter, the massaccumulation efficiency onto the surface of the WD with differential rotation needs more detailed numerical simulations. In this paper, we adopted a simple procedure, whereas the actual process of the thermonuclear burning on the surface of the WDs is likely very complicated. However, the He-star evolutionary channel may play an important role in the formation of brighter $\mathrm{SNe}$ Ia, which are more frequent in the young stellar population (Hamuy et al. 1995; Hamuy et al. 1996; Aubourg et al. 2008). We expect more detailed multi-waveband observations for the companions of SNe Ia to confirm the He-star evolutionary channel in the future, though it is predicted that SNe Ia from this channel may be rare (Kato et al. 2008).

Acknowledgements. We are grateful to the anonymous referee for his/her constructive suggestions that improved this manuscript. This work was supported by the Natural Science Foundation of China (NSFC) under grant number 10873011, China Postdoctoral Science Foundation funded project, and Program for Science \& Technology Innovation Talents in Universities of Henan Province, China. Z.H. thanks the support of NSFC (Grant No. 10821061), the Chinese Academy of Sciences (Grant No. KJCX2-YW-T24) and the 973 project (Grant No. 2007CB815406).

\section{References}

Aldering, G., Goldhaber, G., Perlmutter, S., et al., 1999, ApJ, 517, 565 Aldering, G., Antilogus, P., Scalzo, R. A., et al. 2010, ApJ, 713, 1073 Alexander, D. R., \& Ferguson, J. W. 1994, ApJ, 437, 879 Aubourg, E., Tojeiro, R., Jimenez, R., et al. 2008, A\&A, 492, 631

Chen, W.-C., \& Li, X.-D. 2007, ApJ, 658, L51

Chen, W.-C., \& Li, X.-D. 2009, ApJ, 702, 686

Dewi, J. D. M., Pols O. R., Savonije, G. J., \& van den Heuvel, E. P. J. 2002, MNRAS, 331, 1027

Di Stefano, R., \& Kong, A. K. H. 2003, ApJ, 592, 884

Domínguez, I., Straniero, O., Tornambè, A., \& Isern, J. 1996, ApJ, 472, 783

Domínguez, I., Piersanti, L., Bravo, E., et al. 2006, ApJ, 644, 21

Durisen, R. H. 1977, ApJ, 213, 145

Eggleton, P. P. 1971, MNRAS, 151, 351

Eggleton, P. P. 1972, MNRAS, 156, 361 
A\&A 523, A3 (2010)

Eggleton, P. P. 1973, MNRAS, 163, 279

Filippenko, A. V., Challis, P., Riess, A. G., et al., 1998, AJ, 116, 1009 Garnavich, P. M., Prieto, J. L., \& Han, Z. 2008, ApJ, 677, L109

Guy, J., Regnault, N., Astier, P., et al. 2006, A\&A, 447, 31

Hachisu, I., Kato, M., \& Nomoto, K. 1996, ApJ, 470, L97

Hachisu, I., Kato, M., Nomoto, K., \& Umeda, H. 1999a, ApJ, 519, 314

Hachisu, I., Kato, M., \& Nomoto, K. 1999b, ApJ, 522, 487

Hamuy, M., Phillips, M. M., Maza, J., et al. 1995, AJ, 109, 1

Hamuy, M., Phillips, M. M., Suntzeff, N. B., et al. 1996, AJ, 112, 2391

Han, Z., \& Podsiadlowski, P. 2004, MNRAS, 350, 1301

Han, Z., \& Podsiadlowski, Ph. 2006, MNRAS, 368, 1095

Han, Z., Podsiadlowski, P., \& Eggleton, P. P. 1994, MNRAS, 270, 121

Hicken, M., Garnavich, P. M., Prieto, J. L., et al. 2007, ApJ, 669, L17

Hillebrandt, W., \& Niemeyer, J, C. 2000, ARA\&A, 38, 191

Hillebrandt, W., Sim, S. A., \& Röpke, F. K. 2007, A\&A, 465, L17

Howell, D. A. 2001, ApJ, 554, L193

Hurley, J. R., Pols, O. R., \& Tout, C. A. 2000, MNRAS, 315, 543

Hurley, J. R., Tout, C. A., \& Pols, O. R. 2002, MNRAS, 329, 897

Iben, I. Jr., \& Tutukov, A. V. 1984, ApJS, 54, 335

Iben, I. Jr., \& Tutukov, A. V. 1987, ApJ, 313, 727

Iben, I. Jr., \& Tutukov, A. V. 1991, ApJ, 370, 615

Iben, I. Jr., \& Tutukov, A. V. 1994, ApJ, 431, 264

Iben, I. Jr., Nomoto, K., Tornambè, A., \& Tutukov, A. V. 1987, ApJ, 317, 717

Kahabka, P., \& van den Heuvel, E. P. J., 1997, ARA\&A, 35, 69

Kato, M., \& Hachisu, I. 1994, ApJ, 437, 802

Kato, M., \& Hachisu, I. 2004, ApJ, 613, L129

Kato, M., Hachisu I., Kiyota S., \& Saio H. 2008, ApJ, 684, 1366

Kawabata, K. S., Yamanaka, M., Tanaka, M., et al. 2010, ApJ, 714, 1209

Langer, N., Deutschmann, A., Wellstein, S., \& Höflich, P. 2000, A\&A, 362, 1046

Li, X.-D., \& van den Heuvel, E. P. J. 1997, A\&A, 322, L9

Limongi, M., \& Tornambe, A. 1991, ApJ, 371, 317

Lü, G., Zhu, C., Wang, Z., \& Wang, N. 2009, MNRAS, 396, 1086

Meng, X., \& Yang, W. 2010a, MNRAS, 401, 1118

Meng, X., \& Yang, W. 2010b, ApJ, 710, 1310
Meng, X., Chen, X., \& Han, Z. 2008, A\&A, 487, 625

Meng, X., Chen, X., \& Han, Z. 2009, MNRAS, 395, 2103

Mereghetti, S., Tiengo, A., Esposito, P., et al. 2009, Science, 325, 1222

Narayan, R., \& Popham, R. 1989, ApJ, 346, L25

Nomoto, K. 1982, ApJ, 253, 798

Nomoto, K., Iwamoto, K., \& Kishimoto, N. 1997, Science, 276, 1378

Piersanti, L., Gagliardi, S., Iben, I. Jr., \& Tornambe, A. 2003, ApJ, 598, 1229

Podsiadlowski, P., Mazzali, P., Lesaffre, P., Han, Z., \& Forster, F. 2008, NewAR, 52, 381

Pols, O. R., Tout, C. A., Eggleton, P. P., \& Han, Z. 1995, MNRAS, 274, 964

Pols, O. R., Schroder, K. P., Hurly, J. R., \& Tout, C. A. 1998, MNRAS, 298, 525

Ritter, H. 1985, A\&A, 148, 207

Rogers, F. J., \& Iglesias, C. A. 1992, ApJS, 79, 507

Röpke, F. K., \& Hillebrandt, W. 2005, A\&A, 431, 635

Silverman, J. M., et al. 2010, MNRAS, in press [arXiv: 1003.2417]

Sullivan, M., Nugent, P. E., Howell, D. A., et al., 2006, Nature, 443, 308

Tornambè, A., \& Matteucci, F. 1986, MNRAS, 223, 69

Tutukov, A. V., \& Yungelson, L. R. 1994, MNRAS, 268, 871

Wang, B., \& Han, Z. 2009, A\&A, 508, L27

Wang, B., \& Han, Z. 2010a, A\&A, 515, A88

Wang, B., \& Han, Z. 2010b, RAA, 10, 681

Wang, B., Meng, X., Wang, X., \& Han, Z. 2008, ChJAA, 8, 71

Wang, B., Meng, X., Chen, X., \& Han, Z. 2009a, MNRAS, 395, 847

Wang, B., Chen, X., Meng, X., \& Han, Z. 2009b, ApJ, 701, 1540

Wang, B., Li, X.-D., \& Han, Z. 2010, MNRAS, 401, 2729

Webbink, R. F. 1984, ApJ, 277, 355

Whelan, J., \& Iban, I. Jr. 1973, ApJ, 186, 1007

Woosley, S. E., Taam, R. E., \& Weaver, T. A. 1986, ApJ, 301, 601

Woudt, P. A., Steeghs, D., Karovska, M., et al. 2009, ApJ, 706, 738

Yoon, S. -C., \& Langer, N. 2003, A\&A, 412, L53

Yoon, S. -C., \& Langer, N. 2004, A\&A, 419, 623

Yoon, S. -C., \& Langer, N. 2005, A\&A, 435, 967

Yoon, S. -C., Langer, N., \& Scheithauer, S. 2004, A\&A, 425, 217

Yuan, R. F., Akerlof, C., Miller, J., et al. 2007, Astron. Telegr., 1212, 1 